\title{
ОРГАНИЗАЦИЯ РАБОТЫ
}

УДК 655.4.5+026

А. А. Крулёв

Крыловский государственный научный иентр, Санкт-Петербург

\section{Ведомственное издательство и научно-техническая библиотека в составе единого подразделения}

\begin{abstract}
Освещено объединение научно-технической библиотеки и редакционно-издательского отдела Крыловского государственного научного центра в одно подразделение Информационно-издательский центр. Отмечено, что качественное улучшение деятельности обеих структур по ряду показателей убедительно доказывает целесообразность такой практики. Акцентировано внимание не на процессе объединения, а на достигнутых результатах. Рекомендации по объединению издательства и НТБ адресованы в том числе и руководству различных предприятий и высших учебных заведений, так как представленные мероприятия могут быть реализованы независимо от направления деятельности или профиля подготовки специалистов.
\end{abstract}

Ключевые слова: научно-техническая библиотека, издательство, цитирование, тезаурус, презентация, информационное обслуживание, Крыловский государственный научный центр.

UDC $655.4 .5+026$

Andrey Krulev

Krylov State Research Center, St. Petersburg, Russia

\section{The departmental publisher's and sci-tech library within the single division}

Integrating the Sci-tech library and the Editorial and Publishing Department of Krylov State Research Center into a single division - The Information and Publishing Center is discussed. The author emphasizes that the efficiency of this solution is proved by several improved indicators. He focuses rather on the achievements than on the integration process itself. He also offers his recommendations on integrating publishing services and sci-tech library resources to top managers of organizations and universities and argues that the accomplished integration procedure is applicable to any institutions or professional training area.

Keywords: sci-tech library, publishing house, citation, thesaurus, information services. 
The corporate library of the Krylov State Research Center for shipbuilding exists for more than 120 years. Researchers of the center have been regularly published in the "Shipbuilding" publishing house. Now these two units - the library and the publishing house are merged into a single Information and Publishing Center. We consider the benefits in the chain "publisher-library-reader", that were brought by this merge. The main reason for combining these two successful operating units is the development of electronic communication. Methods of indexing by keywords became available for employees of the publishing house. Consultation with the authors of publications while preparation for release accelerate the process, and allows effectively use refillable thesaurus. Editors receive a deeper knowledge of the thematic areas of the publications issued since the thesaurus helps to better navigate in the existing collections documents and make comparisons. In turn, the bibliography does not spend time on the processing of internal publications and can concentrate on processing the incoming external documents. This is important because such documents are much more difficult to be indexed using the internal thesaurus. Another joint area of collaboration is an analysis of the citations. The library provides consulting support to the bibliometric analyses. To summarize briefly the combined work of librarians and publishers this is the result of combining the two units: 1 . Efficiency and control the transmission of copies of new publications in the library collections. 2. Collaboration in indexing of published materials, increasing the competence of the editors, achievement more active dialogue between the authors and editors at the stage of preparation of the manuscript for publication. 3. Advising authors in the field of rating sources, as well as providing information on the relevance of subjects of published material on the basis of analysis of global databases. This information can be made available to representatives of the academic council for policy in the field of publishing. 4. Presentations in a specially equipped library premises. 5. Release of information products with the involvement of professionals in the field of pre-press editing.

И в вузах, и в научно-исследовательских организациях, в составе которых есть НТБ, - своя специфика организационной структуры. Поэтому речь в статье пойдёт в основном о результатах, достигнутых после объединения этих подразделений в единый Информационно-издательский центр (ИИЦ).

НТБ ФГУП «Крыловский государственный научный центр» (далее Крыловский центр) существует столько же, сколько и сам центр - вот уже более 120 лет. За долгие годы менялись и состав справочно-информационного фонда, и численность персонала, и подчинённость подразделения, однако функции НТБ в целом оставались неизменными, а перечень услуг 
менялся в зависимости от финансовых возможностей Крыловского центра и запросов специалистов.

Издательская деятельность - один из традиционных участков работы Крыловского центра: его сотрудники регулярно публиковались, в первую очередь в издательстве «Судостроение». Существовало ведомственное издательство, выполняющее весь комплекс работ по выпуску научно-технической литературы.

Основная причина объединения этих двух успешно функционирующих подразделений - развитие средств электронной коммуникации.

Н. В. Соколова на одной из конференций РБА говорила о неизбежных изменениях в работе библиотек и издательств: взаимодействие (например, создание классификационных индексов предстоящих публикаций) существовало всегда, а в условиях развития информационных технологий оно расширится за счёт создания интегрированных информационных систем. Работа в таких системах позволит не только автоматизировать и ускорить коммуникационные процессы, но и совершать их максимально открыто для всех участников [1]. Открытость взаимодействия в цепочке «издательство библиотека - читатель» делает этот процесс максимально объективным. Слияние первых двух участников цепочки - не универсальный, но весьма эффективный способ оптимизировать процесс взаимодействия.

Прежде чем говорить о расширении взаимодействия, обусловленного в том числе техническим прогрессом, который меняет и издательскую деятельность, и библиотечно-библиографические процессы, следует обозначить его традиционные участки.

Всем публикуемым материалам, включая научные статьи, сотрудники НТБ присваивали классификационные индексы. По факту издания книги или сборника статей сигнальные экземпляры направлялись в фонд, а получающие их библиографы создавали библиографические записи. Этот порядок работы изменился в связи с созданием электронной библиотеки.

ЭБ Крыловского центра - это информационная система, предназначенная для хранения электронных документов и обеспечивающая доступ к ним через локальную сеть предприятия. Работа с ЭБ ведётся в браузере. С помощью этого сервиса доступны следующие электронные ресурсы: электронный каталог НТБ; электронная коллекция (полнотекстовые электронные документы, обладающие однотипными формальными признаками и содержащие фактографическую информацию); актуализируемый перечень периодических изданий, поступающих в НТБ по подписке; раздел «Новости», в котором регулярно обновляются сведения об изданиях, размещаются интерактивные ссылки на электронные ресурсы [2. С. 29]. 
В НТБ внедрена АБИС «Руслан», благодаря чему все библиотечнобиблиографические процессы автоматизированы. На её базе функционирует ЭБ, предоставляющая пользователям локальной сети возможность доступа не только к ЭК, но и к электронным документам, включая полнотекстовые [2. C. 28].

АБИС пришла на смену внедрённой в 1990-е гг. программе MARC, которая также позволяла создавать ЭК, однако доступ к нему был только у специалистов НТБ. Читатели не могли самостоятельно пользоваться этим каталогом для поиска документов, что приводило к издержкам как в области создания библиографических записей, так и в области их предметизации.

Сегодня, когда доступ к ЭК и полнотекстовым электронным документам возможен с рабочего места, требования читателя к системе поиска неизбежно растут. При индексировании нельзя руководствоваться только личным опытом. Если раньше библиограф в лучшем случае разово консультировался с представителями подразделения, то теперь этот процесс стал более открытым, а следовательно, и объективным. Создано методическое пособие, которое доступно всем в печатном и электронном виде. Оно представляет собой справочник терминов - информационно-поисковый тезаурус.

Обязанности по предметизации, безусловно, не возлагаются на авторов или читателей. Но в новых условиях возможен и актуален активный диалог, а мнение специалиста, который является в то же время и читателем, очень важно.

Методика индексирования ключевыми словами стала доступна и для сотрудников издательства. Консультация с авторами на стадии подготовки издания к выпуску позволяет не только ускорить работу (вместе с документом библиографы получают выбранные автором совместно с редактором рубрики, ключевые слова и т.д.), но и максимально объективно использовать пополняемый тезаурус. Без преувеличения можно сказать, что редакторы получают более глубокие знания в тематических областях издаваемых публикаций, поскольку тезаурус помогает лучше ориентироваться в фонде документов и проводить сопоставления.

В свою очередь, библиографы не тратят время на обработку внутренних изданий и могут сконцентрироваться на работе с поступающими внешними документами. Это важно, поскольку такие документы значительно сложнее индексировать, используя внутренний тезаурус.

Ещё один совместный участок работы - анализ цитируемости публикаций. Это направление, коснувшееся работников вузов в большей степени, чем работников научно-исследовательских организаций, - сложное, но в то 
же время одно из самых перспективных в деятельности и издательств, и библиотек. Многие НТБ не только оказывают консультационную поддержку в области наукометрии и библиометрии, но и включают эти услуги в свои обязанности. А издатели могут очень эффективно использовать сведения о цитируемости, предоставляемые библиотекарями.

Новые возможности таких международных баз цитирования, как Scopus и Web of Science, позволяют пользователям оценить цитируемость публикации и получить сведения о числе публикаций по выбранной теме в мировых высокорейтинговых научных журналах. Издатель не может в полной мере взять на себя ответственность за принятие решений относительно выпуска того или иного издания. Очевидно, что такие решения - в зоне ответственности учёного совета или редакционной коллегии. Но, получая объективные данные, подтверждающие актуальность публикуемых материалов, издатель в глазах руководства может выступать не просто исполнителем, но и полноправным участником обсуждения, в результате которого утверждается издательская политика организации.

С этой точки зрения также очень важны сведения об использовании литературы, имеющейся в фонде и доставляемой по МБА. При должном анализе издательство может обоснованно настаивать на корректировке плана выпуска продукции.

Необходимо сказать несколько слов о поступлении документов в фонд НТБ. Из личного опыта знаю, что вопросы, задаваемые библиотекарям (различных профильных организаций) относительно новинок изданий, выпускаемых учреждением, в состав которого входят НТБ, порой вызывают замешательство. И это далеко не всегда связано с техническими аспектами (отсутствие ЭК и т.п.). К сожалению, библиотекарь нередко не знает о том, какая литература вышла или скоро выйдет в ведомственном издательстве. Был случай, когда научная литература, выпущенная в одном из вузов, поступила в фонд НТБ Крыловского центра раньше, чем в фонд библиотеки того вуза.

На вопрос о возможностях использования полнотекстовых материалов в ЭБ сотрудники библиотек зачастую тоже не могут оперативно ответить, поскольку оформлением нормативных документов, регламентирующих порядок работы по электронной доставке и т.д., занимаются издатели.

Справедливости ради нужно сказать, что представители издательств также часто не обладают информацией о судьбе выпускаемой ими продукции. Вопросы о том, как получить то или иное издание, нередко остаются без ответов.

Сводить функции издательства только к процессу подготовки рукопи- 
си к печати и руководству полиграфическим производством означает отказываться от анализа того, как используется издаваемая литература. Это, в свою очередь, значительно обесценивает роль издательского подразделения в организации, особенно когда нет прямой коммерческой составляющей. В этом случае вполне объяснимо решение руководства обратиться к контрагенту.

Ещё один положительный пример совместной деятельности НТБ и издательского центра - организация и проведение презентаций вышедших в свет изданий. Такие мероприятия, как правило, проходят в читальном зале НТБ, оснащённом современным демонстрационным оборудованием. Не исключаю, что презентации можно проводить и непосредственно в издательстве, однако это, скорее, исключение, чем правило.

Очевидное преимущество таких мероприятий - возможность пообщаться с автором, получить экземпляр издания с автографом. Именно благодаря подобным встречам может состояться настоящий диалог, поскольку в одном помещении собираются не просто коллеги, а учёные, проявившие профессиональный интерес к выпущенной книге. Нередко презентация проводится в формате, близком к конференции. Не будет преувеличением сказать, что такие мероприятия наилучшим образом раскрыли возможности ведомственной НТБ как места для подобных встреч.

N. Kranich, M. Lotts и G. Springs называют университетскую библиотеку местом, где члены университетского сообщества могут общаться без границ, в спокойной атмосфере [3]. Основной перспективной задачей библиотеки авторы считают именно организацию пространства для коммуникаций. Важно отметить: несмотря на развитие электронных ресурсов, библиотека должна сохранить свой статус места встречи, диалога читателя и библиотекаря. В России многие университетские библиотеки сохранили этот статус, однако в силу разных причин это направление незаслуженно забыто, а работа НТБ сводится к хранению документов и организации доступа к ним.

В Крыловском центре НТБ сегодня - практически единственное место, где все сотрудники могут собраться для обсуждения любого вопроса. В отличие от тематических конференций, совещаний с жёстким регламентом и ограниченным количеством участников, встречи в библиотеке, которые организовать гораздо проще, становятся наиболее предпочтительными мероприятиями для сотрудников. Такие мероприятия полезны и для представителей издательства: они могут узнать реакцию читателей на новое издание, проанализировав число посетителей, активность дискуссии и т.п. 
Одно из главных преимуществ слияния в единое подразделение - выпуск библиотекой собственных информационных продуктов, в том числе в печатном виде. Благодаря совместному труду библиотекарей и издателей сотрудники регулярно получают информационные бюллетени о новых поступлениях в НТБ и другие печатные продукты. Они прекрасно дизайнерски и полиграфически оформлены, что имеет весьма существенное значение. Раньше библиотекарь, не имеющий опыта в области вёрстки, дизайна и полиграфии, готовил по запросу клиента библиографическую справку в текстовом редакторе.

Кто-то может возразить: перечисленные направления работы (передача документов от издателей в библиотеку, проведение мероприятий, выпуск информационной продукции) можно развивать и без объединения подразделений. Однако опыт показывает, что именно единоначалие лучшим образом способствует организации работы, в том числе и потому, что издержки, связанные с координацией деятельности сотрудников (консультации и т.п.), сведены к минимуму.

Подводя итоги, кратко перечислим новые возможности совместной деятельности, которые появились в результате объединения двух подразделений.

Оперативность и контроль при передаче экземпляров новых изданий в фонд НТБ. Эта работа способствует установлению более тесных связей с коллегами из смежных организаций благодаря оповещениям.

Совместная работа по предметизации публикуемых материалов, повышение компетенции редакторов, более активный диалог с авторами уже на стадии подготовки рукописи к изданию. Оперативное создание библиографических записей.

Консультирование авторов в области рейтинговой оценки используемых источников, а также предоставление сведений об актуальности тематики публикуемых материалов на основе анализа мировых баз научного цитирования. Эти сведения могут быть предоставлены представителям учёного совета для определения политики в издательской деятельности.

Проведение презентаций в специально оборудованном помещении НТБ. Единоначалие в этой деятельности (как и в остальных случаях) не является определяющим условием, но помогает свети к минимуму организационные издержки.

Выпуск информационных продуктов с привлечением профессионалов в области допечатной подготовки и полиграфии.

Выгоды от совместной, а точнее единоначальной деятельности не исчерпываются перечисленными возможностями. В статье представлены 
только некоторые из тех направлений работы, которые уже успешно реализуются в ИИЦ Крыловского центра.

Непрерывный рост средств электронной коммуникации, увеличение количества электронных документов, другими словами - технический прогресс, диктуют новые условия работы и издательствам, и библиотекам, основным объектом деятельности которых на протяжении веков была печатная книга. Происходящие процессы не могут не сказаться и на управлении этими структурами. Возможно, объединение - это не только способ повысить эффективность деятельности, но и единственное средство выжить в новую эпоху.

\section{СПИСОК ИСТОЧнИков}

1. Соколова Н. В. Библиотеки и издательства - новые формы сотрудничества в едином информационном пространстве / Н. В. Соколова. - Режим доступа: http://www.rba.ru/content/ activities/section/12/mag/mag08/23.pdf.

Sokolova $\mathbf{N}$. V. Biblioteki i izdatelstva - novye formy sotrudnichestva v edinom informatsionnom prostranstve /N. V. Sokolova.

2. Крулёв А. А. Системный рубрикатор и тезаурус ведомственной технической библиотеки: особенности, возможности и перспективы / А. А. Крулёв // НТИ. Сер. 2. - 2016. - № 3. C. $28-33$.

Krulev A. A. Sistemnyy rubrikator i tezaurus vedomstvennoy tehnicheskoy biblioteki: osobennosti, vozmozhnosti i perspektivy/A. A. Krulev // NTI. Ser. 2. - 2016. - № 3. - S. 28-33.

3. Kranich N. The promise of academic libraries / N. Kranich, M. Lotts, G. Springs // College and Research Libraries News. - 2014. - Vol. 75 - № 4. - P. 182-186.

Andrey Krulev, Head, Sci-tech Information Processing Group, Krylov State Research Center;

aa_krulev@ksrc.ru

44, Moskovskoye sh., 196158 St. Petersburg, Russia 